\title{
Larvas de Scinax rizibilis (Bokermann) (Anura, Hylidae): avaliando o efeito parental
}

\author{
Rogério P. Bastos ${ }^{1}$ \\ Célio F.B. Haddad ${ }^{2}$
}

\begin{abstract}
Tadpoles of Scinax rizibilis (Bokermann) (Anura, Hylidae): evaluating the paternal effects. A controlled experiment, related with the mating system of Scinax rizibilis (Bokermann, 1964), was conducted to assess if larval variation could be due to size of male or its ability to manage an amplexus. Adult individuals were caught during breeding activity (from February 1993 to January 1994), in a temporary pond in the municipality of Ribeirão Branco, São Paulo State, southeastern Brazil. The duration of the larval period was not different between tadpoles of large and small males, nor was it different between tadpoles coming from natural or artificial pairs. The reproductive status of the male (if it had managed an amplexus) also did not influence the total length nor the mass of the tadpoles close to metamorphosis. However, tadpoles of larger and heavier males were, on average, approximately $5.5 \%$ and $11 \%$ larger and heavier, respectively, than tadpoles of smaller males. These results indicate that the breeding system of $S$. rizibilis could potentially have a directional effect on the larval characteristics.
\end{abstract}

KEY WORDS. Scinax rizibilis, mating system, larval characteristic

Os processos e as conseqüências decorrentes dos sistemas de acasalamento têm proporcionado diversos estudos tanto empíricos como teóricos (WILCZYNISKI \& RYAN 1992; WAGNER 1998). A manutenção, pela seleção natural, de preferências reprodutivas pelas fêmeas é raramente questionada para espécies nas quais os machos de anuros fornecem recursos ou realizam cuidado parental (veja exemplos em HOWARD 1978; KLUGE 1981). Por outro lado, quando os machos não fornecem nem recursos nem cuidado parental, a manutenção de preferências reprodutivas é mais controversa (WOODWARD 1987; HowARD et al. 1994). Todavia, estudos recentes, com espécies que apresentam estas características reprodutivas, têm demonstrado que a escolha realizada pela fêmea é adaptativa (BOURNE 1993; BASTOS \& HADDAD 1996).

Os anuros são excelentes objetos de estudo dos efeitos evolutivos do sistema de acasalamento, pois é possível identificar diversas características que afetam a performance de girinos, tais como crescimento larval mais rápido (TRAVIS 1983) e período larval menor (TRAVIS \& TREXLER 1986). A comparação entre as características dos girinos poderia permitir avaliar se as dimensões do macho (massa ou comprimento rostro-anal) ou a sua capacidade de conseguir amplexo são relacionadas com a performance larval, de maneira que se possa verificar se há alguma vantagem para a descendência.

1) Departamento de Biologia Geral, Instituto de Ciências Biológicas, Universidade Federal de Goiás. Caixa Postal 131, 74001-970 Goiânia, Goiás, Brasil. E-mail: bastos@icb1.ufg.br

2) Departamento de Zoologia, Instituto de Biociências, Universidade Estadual Paulista. Caixa Postal 199, 13506-900 Rio Claro, São Paulo, Brasil. E-mail: haddad@ms.rc.unesp.br

Revta bras. Zool. 18 (4): 1127 - 1133, 2001 
Scinax rizibilis (Bokermann, 1964) é um hilídeo pertencente ao grupo catharinae, encontrado na região leste do Brasil (Frost 1985), em cujo sistema de acasalamento pode ser observado tanto vantagem reprodutiva dos machos mais pesados como acasalamentos por classes de CRA (comprimento rostro-anal) ou massa (BASTOS \& HADDAD 1999). Assim, qual seria a vantagem de fêmeas acasalarem com determinados machos?

O objetivo principal deste trabalho foi verificar como o sistema de acasalamento, considerando as características paternas de tamanho (CRA ou massa) ou capacidade de conseguir amplexo, poderiam influenciar as características larvais (comprimento total, massa e duração do período larval) de Scinax rizibilis (Hylidae).

\section{MATERIAL E MÉTODOS}

As características dos girinos foram avaliadas através da descendência de quatro fêmeas, capturadas em amplexo, na poça localizada na Fazendinha São Luiz ( $24^{\circ} 21^{\prime}$ 'S , 48 $44^{\prime}$ 'W), município de Ribeirão Branco, São Paulo, Brasil. Essas fêmeas foram cruzadas cada uma com quatro machos diferentes (Tab. I). Os cruzamentos obtidos para cada fêmea foram os seguintes: (1) fêmea com macho original em amplexo (casal natural); (2) fêmea com outro macho encontrado em amplexo; fêmea com machos que não obtiveram amplexo, sendo (3) um com CRA e massa menores que a média geral da população e (4) outro com CRA e massa maiores que a média da população (veja BASTOS \& HADDAD 1999, para os valores médios de CRA). Os três últimos pares formados foram denominados de casais artificiais.

Tabela I. Cruzamentos realizados entre fêmeas e machos de Scinax rizibilis (indicados por X) para a avaliação do efeito sobre características larvárias. Para a designação do status reprodutivo do macho: (S) solitário; (A) em amplexo. Para a categoria de CRA dos machos: (P) pequeno; $(G)$ grande. Os machos estão também indicados com as fêmeas com que se encontravam em amplexo.

\begin{tabular}{|c|c|c|c|c|c|c|c|}
\hline \multirow[t]{2}{*}{ Machos } & \multirow[t]{2}{*}{ Status reprodutivo } & \multirow{2}{*}{$\begin{array}{l}\text { Mecho em amplexo } \\
\text { com fêmea }\end{array}$} & \multirow{2}{*}{$\begin{array}{l}\text { Categoria } \\
\text { de CRA }\end{array}$} & \multicolumn{4}{|c|}{ Fêmeas } \\
\hline & & & & 1 & 2 & 3 & 4 \\
\hline 1 & A & 1 & G & $x$ & $x$ & & \\
\hline 2 & A & 2 & $\mathrm{P}$ & $x$ & $x$ & & \\
\hline 3 & $\mathrm{~s}$ & & $P$ & $x$ & & & \\
\hline 4 & $\mathrm{~s}$ & & G & $x$ & & & \\
\hline 5 & $\mathrm{~s}$ & & $\mathrm{P}$ & & $x$ & & \\
\hline 6 & $\mathrm{~s}$ & & G & & $\mathrm{x}$ & & \\
\hline 7 & A & 3 & G & & & $x$ & $\mathrm{x}$ \\
\hline 8 & A & 4 & $\mathrm{P}$ & & & $x$ & $\mathrm{x}$ \\
\hline 9 & $\mathrm{~s}$ & & $\mathrm{P}$ & & & $x$ & \\
\hline 10 & $\mathrm{~s}$ & & G & & & $\mathrm{x}$ & \\
\hline 11 & $\mathrm{~s}$ & & $\mathrm{P}$ & & & & $x$ \\
\hline 12 & $\mathrm{~s}$ & & G & & & & $\mathrm{x}$ \\
\hline
\end{tabular}

Em laboratório, machos e fêmeas foram medidos com paquímetro (precisão de $0,01 \mathrm{~mm}$ ) e pesados com balança Pesola ${ }^{\circledR}$ (precisão de $0,05 \mathrm{~g}$ ), sendo os machos separados nas categorias pequeno (menor que a média geral de CRA para a população) e grande (maior que a média geral para a população). A fertilização foi realizada banhando os óvulos em suspensões de espermatozóides, seguindo o 
procedimento de TRAVIS $(1980,1981)$. Para cada macho de S. rizibilis foram preparadas duas soluções aquosas com espermatozóides, nas quais adicionou-se os óvulos, que foram obtidos através de compressão manual dos flancos das fêmeas.

Quando todos os ovos eclodiram, oito girinos foram escolhidos aleatoriamente de cada uma das 16 coortes obtidas. Todos os girinos foram mantidos individualmente em recipientes de $300 \mathrm{ml}$ de água sem cloro. Os recipientes foram acondicionados, lado a lado, em uma única bancada de um laboratório com temperatura do ar constante a $25^{\circ} \mathrm{C}$. Cada girino foi alimentado com ração para peixes ornamentais a partir do terceiro dia após a eclosão. A ração foi oferecida a cada três dias, sendo que antes os recipientes tiveram a água trocada. A quantidade de ração foi aumentada gradativamente (de 10 $\mathrm{mg} /$ dia a $20 \mathrm{mg} /$ dia), até o final do experimento.

Os girinos foram medidos (comprimento total) com paquímetro (precisão de $0,01 \mathrm{~mm}$ ) e pesados com balança analítica (com precisão de $0,0001 \mathrm{~g}$ ), quando se aproximaram da metamorfose (estágio 42 de GOSNER 1960).

Os girinos obtidos pelos diversos cruzamentos foram divididos em quatros grupos: (1) os de machos maiores, (2) os de machos menores, (3) girinos de casais naturais e (4) girinos de casais artificiais, para posterior comparação das características larvais. Para a análise estatística foi utilizada a análise de variância (ANOVA) fatorial (SOKAL \& RHOLF 1994) e o teste " $\mathrm{t}$ " (ZAR 1996). As estatísticas "F" foram calculadas para se testar os efeitos dos cruzamentos realizados sobre as características larvais. Um nível de significância $\mathrm{P}<0,05$ foi utilizado.

\section{RESULTADOS}

Os machos que obtiveram amplexo $(\mathrm{n}=4)$ não apresentaram valores médios de CRA $(26,88 \pm 1,21 \mathrm{~mm})$ e de massa $(0,89 \pm 0,09 \mathrm{~g})$ significativamente diferentes $\left(\mathrm{t}_{\text {cra }}=0,26 ; \mathrm{gl}=7 ; \mathrm{p}>0,05 ; \mathrm{t}_{\text {massa }}=1,22 ; \mathrm{gl}=7 ; \mathrm{p}>0,05\right)$ dos valores médios de CRA $(26,67 \pm 1,29 \mathrm{~mm})$ e de massa $(0,82 \pm 0,11 \mathrm{~g})$ dos machos que não conseguiram amplexo $(n=8)$.

Entre as três características larvais analisadas, o comprimento total foi a que menos apresentou variação (Tabs II, III). A média dos comprimentos totais variou, entre todas as coortes, de 27,13 a 31,56 mm (Tab. II). Os girinos dos casais artificiais foram cerca de 2,3\% maiores, porém não significativamente, que os girinos produzidos pelos casais naturais (Tabs III, IV). Os girinos de machos grandes foram cerca de $5,5 \%$ maiores que os girinos de machos pequenos, sendo essa diferença significativa (Tab. IV).

A média das massas dos girinos, próximos ao estágio 42, variou de 181,0 a $277,9 \mathrm{mg}$ entre todas as coortes (Tab. II). Os girinos dos casais artificiais foram cerca de 3,1\% mais pesados, porém não significativamente, do que os girinos produzidos pelos casais naturais (Tabs III, IV). Os girinos de machos grandes foram cerca de $11 \%$ mais pesados que os girinos de machos pequenos, sendo essa diferença significativa (Tab. IV).

A duração do período larvário variou, entre todas as coortes (Tab. II), de 23 a 33 dias. Todavia, não houve diferença significativa entre a duração do período larvário de girinos de machos maiores quando comparados aos de machos menores ou entre os girinos de casais naturais e artificiais (Tabs III, IV). 
Tabela II. Valores médios das características larvais de Scinax rizibilis como uma função do tipo de acasalamento. (A) Acasalamento com macho original; (B) com outro macho que estava em amplexo; (C) com macho pequeno; (D) com macho grande.

\begin{tabular}{lrrrrr}
\hline \multirow{2}{*}{ Caracteristicas } & \multirow{2}{*}{ Tipo de acasalamento } & \multicolumn{4}{c}{ Fêmeas } \\
\cline { 3 - 6 } & & 1 & 2 & 3 & 4 \\
\hline Comprimento total $(\mathrm{mm})$ & A & 31,39 & 29,29 & 29,31 & 27,13 \\
& B & 27,93 & 32,14 & 30,04 & 29,46 \\
& C & 29,17 & 31,11 & 29,24 & 28,73 \\
& D & 29,95 & 31,56 & 30,64 & 30,96 \\
\hline Massa (m) & A & 230,30 & 219,70 & 250,10 & 181,00 \\
& B & 189,70 & 238,30 & 247,80 & 227,10 \\
& C & 202,70 & 232,60 & 229,10 & 213,30 \\
& D & 215,30 & 218,60 & 277,90 & 229,60 \\
\hline Duraçäo do período larval (dias) & A & 24,50 & 24,38 & 31,43 & 30,14 \\
& B & 23,13 & 25,25 & 32,57 & 30,43 \\
& C & 24,43 & 27,00 & 30,43 & 28,43 \\
& D & 23,83 & 23,29 & 30,75 & 29,38 \\
\hline
\end{tabular}

Tabela III. Valores médios das características larvais para cada grupo de girinos de Scinax rizibilis, Ribeirão Branco, São Paulo.

\begin{tabular}{|c|c|c|c|c|}
\hline Caracteristicas larvais & Origem do girino & $n$ & $\mathrm{X} \pm \mathrm{DP}$ & Amplitude \\
\hline \multirow[t]{4}{*}{ Comprimento total (mm) } & Casais naturais & 30 & $29,36 \pm 2,72$ & 23,1 a 34,8 \\
\hline & Casais artificiais & 86 & $30,05 \pm 2,09$ & 24,3 a 34,2 \\
\hline & Machos pequenos & 57 & $29,05 \pm 2,15$ & 23,1 a 32,4 \\
\hline & Machos grandes & 59 & $30,74 \pm 2,09$ & 25,1 a 34,8 \\
\hline \multirow[t]{4}{*}{ Massa (mg) } & Casais naturais & 30 & $220,60 \pm 33,84$ & 156,3 a 302,1 \\
\hline & Casais artificiais & 86 & $227,50 \pm 37,76$ & 144,8 a 333,7 \\
\hline & Machos pequenos & 57 & $214,60 \pm 32,76$ & 144,8 a 298,4 \\
\hline & Machos grandes & 59 & $240,10 \pm 36,54$ & 167,9 a 333,7 \\
\hline \multirow[t]{4}{*}{ Duração do período larval (dias) } & Casais naturais & 30 & $27,37 \pm 3,82$ & 22,0 a 34,0 \\
\hline & Casais artificiais & 86 & $27,51 \pm 3,64$ & 22,0 a 38,0 \\
\hline & Machos pequenos & 57 & $27,42 \pm 3,60$ & 22,0 a 34,0 \\
\hline & Machos grandes & 59 & $27,43 \pm 3,66$ & 22,0 a 38,0 \\
\hline
\end{tabular}

Tabela IV. Análise de variância para as características larvais de Scinax rizibilis. Um nivel de significância de $\mathrm{P}<0,05$ indica que houve diferença entre os girinos de cada tratamento.

\begin{tabular}{|c|c|c|c|c|c|}
\hline Características larvais & Origem do girino & $g \mid$ & Qm & $\mathrm{F}$ & $P$ \\
\hline \multirow[t]{2}{*}{ Comprimento total (mm) } & $\begin{array}{c}\text { Casais naturais } \\
x \\
\text { Casais artificiais } \\
\text { Machos pequenos }\end{array}$ & 1 & 14,1886 & 3,72 & $>0,05$ \\
\hline & $\begin{array}{c}x \\
\text { Machos grandes }\end{array}$ & 1 & 74,8704 & 19,64 & $<0,01$ \\
\hline \multirow[t]{2}{*}{ Massa (mg) } & $\begin{array}{c}\text { Casais naturais } \\
\times \\
\text { Casais artificiais } \\
\text { Machos pequenos }\end{array}$ & 1 & 426,2048 & 0,43 & $>0,05$ \\
\hline & $\begin{array}{c}\mathrm{x} \\
\text { Machos grandes }\end{array}$ & 1 & $9.693,7746$ & 9,89 & $<0,01$ \\
\hline \multirow[t]{2}{*}{ Duração do período larval (dias) } & $\begin{array}{c}\text { Casais naturais } \\
x \\
\text { Casais artificiais } \\
\text { Machos pequenos }\end{array}$ & 1 & - 2,2508 & 0,70 & $>0,05$ \\
\hline & $\begin{array}{c}\mathrm{x} \\
\text { Machos grandes }\end{array}$ & 1 & 0,6618 & 0,21 & $>0,05$ \\
\hline
\end{tabular}




\section{DISCUSSÃO}

Nas agregações reprodutivas de $S$. rizibilis, o sistema de acasalamento é muito variável, podendo ser encontrada tanto reprodução dependente de tamanho ou escolha por classes de machos (CRA e massa de machos e de fêmeas em amplexo correlacionados; BASTOS \& HADDAD 1999), havendo evidências que os machos são selecionados pelas fêmeas.

Os resultados dos experimentos realizados indicam que, potencialmente, o sistema de acasalamento pode afetar as características larvais em S. rizibilis. As diferenças entre as três características larvais das coortes de casais naturais e artificiais de $S$. rizibilis, foram muito pequenas, sendo que nenhuma delas foi significativa. Todavia, girinos originados de machos grandes foram significativamente maiores $(5,5 \%)$ e mais pesados $(11 \%)$ que os girinos originados de machos pequenos. Segundo FALCONER (1975), caracteres morfológicos possuem componente genético mais forte (menos sujeitos a efeitos ambientais) do que caracteres fisiológicos (que refletiriam, por exemplo, na duração do período larval). Assim, os resultados obtidos para $S$. rizibilis parecem estar dentro do esperado pela genética quantitativa.

A constatação da influência parental nos anuros tem ocasionado controvérsias (WoOdward et al. 1988; Mitchell 1990; Howard et al. 1994). Em Bufo woodhousii Girard, 1854, os girinos originados de machos grandes possuíam maior tamanho na metamorfose, que os girinos originados de machos pequenos (MrTCHELL 1990), ao passo que em Pseudacris crucifer (Wied-Neuwied, 1838) apresentavam maior taxa de crescimento (WoodWARD et al. 1988). Todavia, em Bufo americanus Holbrook, 1836 as características larvais não foram correlacionadas nem com o tamanho e nem com a idade dos machos (HowARD et al. 1994).

Para girinos de S. rizibilis poderia ser vantajoso possuírem um tamanho e peso maiores na metamorfose, o que excluiria diversos carnívoros de menor tamanho como predadores potenciais de jovens recém-metamorfoseados desta espécie. Situação similar foi observada para Pseudacris crucifer (TRAVIS et al. 1987). Um tamanho maior na metamorfose pode significar também vantagem em termos metabólicos (PoUGH \& KAMEL 1984), refletindo, então, na vida posterior do girino. Há evidências de que girinos maiores na metamorfose possam originar anuros que se reproduzam mais cedo (SMITH 1987) ou que sejam maiores ao atingirem a maturidade sexual (SMITH 1987; BERVEN 1990).

Talvez um girino de $S$. rizibilis com tamanho e peso maiores na metamorfose se torne um sub-adulto com alguma vantagem inicial sobre o sub-adulto gerado de um girino com tamanho e peso menores. Para Spea multiplicata (Cope, 1863) (Pelobatidae) os juvenis originados de machos maiores ou de casais naturais, apresentaram maior taxa de crescimento nos primeiros 30 dias após a metamorfose (WOODWARD 1986). Todavia, de acordo com RYAN (1991), é difícil saber se estas características larvais refletem, de fato, maior viabilidade ao longo da vida posterior do anuro, pois esta vantagem inicial pode desaparecer com o desenvolvimento posterior, de forma que não haja diferença de viabilidade entre os diferentes adultos originados. 
AGRADECIMENTOS. Gostaríamos de agradecer a J.A.F. Diniz-Filho pela leitura crítica e sugestões apresentadas ao manuscrito. A J.P. Pombal Jr., E.C. P. Pombal, O.C. Oliveira, F. Baldisseri, pelo auxílio nos trabalhos de campo. A A.T. d'Heursel pela ajuda na confecção do abstract. Aos Srs. Newton e Nilson Mattedi pela permissão de acesso ao local de estudos. A D.V. Andrade e M.C. Crupi pela inestimável ajuda durante os experimentos. A FAPESP (proc. 92/0631-2, 94/5634-5 e 95/9269-2) pelo apoio financeiro. Ao CNPq pelas bolsas de produtividade em pesquisa. RPB agradece ao CNPq e CAPES pela bolsa durante o doutoramento na UNESP (campus de Rio Claro-SP).

\section{REFERÊNCIAS BIBLIOGRÁFICAS}

BASTOS, R.P. \& C.F.B. HADDAD. 1996. Breeding activity of the neotropical treefrog Hyla elegans (Anura, Hylidae). Jour. Herpetol. 30 (3): 355-360.

- 1999. Atividade reprodutiva de Scinax rizibilis (Bokermann) (Anura, Hylidae) na Floresta Atlântica, sudeste do Brasil. Revta bras. Zool. 16 (2): 409-421.

BERVEN, K. A. 1990. Factors affecting population fluctuations in larval and adult stages of the wood frog (Rana sylvatica). Ecology 71: 1599-1608.

BournE, G. 1993. Proximate costs and benefits of mate acquisition at leks of the Ololygon rubra. Animal

Behav. 45: 1051-1059.

FALCONER, D.S. 1975. Introduction to quantitative genetics. Kent, Longman, 364p.

Frost, D.R. 1985. Amphibians species of the world. Kansas, Allen Press, 732p.

GoSNER, K. 1960. A simplified table for staging anuran embryos and larvae with notes on identification. Herpetologica 16: 183-190.

How ARD, R.D. 1978. The evolution of mating strategies in bullfrogs, Rana catesbeiana. Evolution 32 (4): 850-871.

Howard, R.D.; H.H. Whiteman \& T. SChueller. 1994. Sexual selection in American toads: a test of a good-genes hypothesis. Evolution 48 (4): 1286-1300.

KLuge, A.G. 1981. The life history, social organization, and parental behavior of Hyla rosenbergi Boulenger, a nest-building giadiator frog. Misc. Publ. Mus. Zool., Univ. Mich., 160: 1-170.

MrTCHELL, S.L. 1990. The mating system genetically affects offspring performance in woodhouse's toad (Bufo woodhousii). Evolution 44: 502-519.

PoUGH, F.H. \& S. KAMEL. 1984. Post-metamorphic change in activityt metabolism of anurans in relation to life history. Oecologia 65: 138-144.

Ryan, M.J. 1991. Sexual selection and communication in frogs. Trends Ecol. Evol. 6 (11): 351-355.

SMITH, D.C. 1987. Adult recruitment in chorus frogs: effects on size and date at metamorphosis. Ecology 68: $344-350$

SoKAL, R.R. \& F.J. RohlF. 1994. Biometry. San Francisco, Freeman and Co., $3^{\text {rd }}$ ed., 887p.

TRAVIS, J. 1980. Genetic variation for larval specific growth rate in the frog Hyla gratiosa. Growth 44: 167-181.

- 1981. The control of larval growth variation in a population of Pseudacris triseriata (Anura: Hylidae). Evolution 35: 423-432.

- 1983. Variation in development patterns of larval anurans in temporary ponds. I. Persistent variation within a Hyla gratiosa population. Evolution 37 (3): 496-512.

TRAVIS, J. \& J.C. TREXLER. 1986. Interactions among factors affecting growth, development and survival in experimental populations of Bufo terrestris (Anura: Bufonidae). Oecologia 69: 110-116.

TRAVIS, J.; S.B. EMERSON \& M. BLOUIN. 1987. A quantitative-genetic analysis of larval life-history traits in Hyla crucifer. Evolution 41 (1): 145-156.

WAGNER JR., W.E. 1998 Measuring female mating preferences. Animal Behav. 55: 1029-1042.

WILCZYNSKI, W. \& M.J. RYAN. 1992. Introduction to the symposium: mechanism of mate choice. Amer. Zool. 32: 15-17.

Revta bras. Zool. 18 (4): 1127 - 1133, 2001 
WoodwARD, B.D. 1986. Paternal effects on juvenile growth in Scaphipus multiplicatus (the New Mexico spadefoot toad). Amer. Nat. 128 (1): 58-65.

- 1987. Paternal effects of offspring traits in Scaphiopus couchi (Anura: Pelobatidae). Oecologia 73: 626-629.

WoOdWARD, B.D.; J. TRAVIS \& S. MITCHELL. 1988. The effects of the mating system progeny perfomance in Hyla crucifer (Anura: Hylidae). Evolution 42 (4): 784-794.

ZAR, J.H. 1996. Biostatistical Analysis. New Jersey, Prentice-Hall, $3^{\text {rd }}$ ed., 662p.

Recebido em 15.XII.2000; aceito em 22.X.2001. 